\title{
Comparación entre cuestionario de control de asma en niños (CAN) y recomendaciones de control GINA
}

\author{
ALBERTO VIDAL G.*, ANA MARÍA ESCOBAR C.* y MARÍA EUGENIA MEDINA R.**
}

\author{
Comparison of asthma control questionnaire in children (CAN) and GINA control \\ recommendations
}

Introduction: Several questionnaires of asthma control exist for children, but few of them have been validated in Spanish being applicable to our country. Patients and Methods: We compared the asthma control questionnaire in children (CAN) in two versions in Spanish with the control questionnaire recommended by GINA. The study was carried out at Roberto del Rio Children's Hospital, Santiago de Chile. We measured concordance and diagnostic tests. The sample size was calculated considering a power of $80 \%$ and a $p$ value $<0,05$. Results: We enrolled to 214 asthmatic children from 5 to 14 yearsold. Male gender $60.3 \%$. Mean of age was 8.3 years. Controlled asthma in children 5 to 8 years-old was $12.1 \%$ for GINA and 35.6\% for CAN guardian version. CAN guardian achieved $76.5 \%$ to agree in uncontrolled asthma (Kappa 0.39). Sensitivity, specificity, positive predictive and negative predictive values for children from 5 to 8 years-old were 100\%, 73\%, 34\% and 100\% respectively. Controlled asthma in children from 9 to 14 years-old reached $23.2 \%$ with GINA and $43.9 \%$ with CAN. The 79.2\% of the controlled asthmatic by GINA were correctly diagnosed by CAN (Kappa 0.55). Sensitivity, specificity, positive predictive and negative predictive values in 9 to 14 years-old children were $100 \%, 73 \%$, $53 \%$ and $100 \%$ respectively. Conclusion: CAN is an instrument of easy implementation in our practice that achieves acceptable agreement with GINA recommendations, whose main utility is in identifying the patients with uncontrolled asthma.

Key words: Asthma control, children, adolescents.

\section{Resumen}

Introducción: Existen varios cuestionarios de control de asma para niños, pero hay pocas versiones validadas en español aplicables a nuestro medio. Pacientes y Métodos: Se comparó un cuestionario de control de asma para niños (CAN) en sus dos versiones en español con el cuestionario de control recomendado por GINA. El estudio fue realizado en el Hospital de niños Roberto del Río. Se midió concordancia y pruebas diagnósticas. Se calculó el tamaño muestral considerando un poder de $80 \%$ $y$ valor de $p<0$,05. Resultados: Se enrolaron al estudio 214 niños asmáticos de 5 a 14 años de edad. 60,3\% de género masculino. Promedio de edad 8,3 años. El asma controlada en niños de 5 a 8 años se encontró en 12,1\% con GINA y 35,6\% con CAN tutor. CAN tutor logró 76,5\% de acuerdo en asma no controlada (Kappa 0,39). La sensibilidad, especificidad, valor predictivo positivo y negativo para niños de 5 a 8 años fue de 100\%, 73,3\%, 34\% y 100\% respectivamente. Asma controlada en niños de 9 a 14 años alcanzó a 23,2\% con GINA y a 43,9\% con CAN niño/adolescente. El 79,2\% de los asmáticos controlados por GINA fueron correctamente diagnosticados por CAN niño/adolescente (Kappa 0,55). La sensibilidad, especificidad, valor predictivo positivo y negativo en niños de 9 a 14 años fue de 100\%, 73\%, 53\% y 100\% respectivamente. Conclusión: CAN es un instrumento de fácil implementación en nuestro medio que logra concordancia aceptable con GINA, cuya mayor utilidad está en identificar a los pacientes con asma no controlada.

Palabras clave: Control de asma, niños, adolescentes.

\footnotetext{
* Pediatra Especialista en Enfermedades Respiratorias Pediátricas, Hospital de niños Roberto del Río.

** Tecnólogo Médico Hospital de niños Roberto del Río.
} 


\section{Introducción}

Los cuestionarios de control de síntomas son ampliamente recomendados por las guías internacionales en la monitorización del control del asma en niños y adultos ${ }^{1-3}$. Los dos más difundidos son el Asthma Control Test (ACT) de Nathan et al., y el Asthma Control Questionnaire (ACQ) de Juniper et al., ambos diseñados para medir el control de asma en pacientes mayores de 12 años y adultos, con versiones validadas en inglés y español ${ }^{4-7}$.

En los últimos años se han publicado una decena de cuestionarios para medir el control de asma en niños menores de 12 años. De estos, cuatro son los más reconocidos: Asthma Therapy Assessment Questionnaire for Children and Adolescents (C-ATAQ), Childhood asthma control test (C-ACT), Asthma Control Questionnaire in children (ACQ) y Cuestionario de control de asma en niños (CAN). C-ATAQ es un cuestionario validado en inglés para niños entre 5 y 17 años, con 6 preguntas dirigidas a los padres o cuidadores ${ }^{8,9}$. C-ACT, validado en inglés para niños de 4 a 11 años, tiene siete preguntas, cuatro de las cuales deben ser respondidas por el niño en una escala visual análoga y tres por su cuidador ${ }^{10}$. ACQ, recientemente validado en inglés por Juniper et al, para niños entre 6 y 16 años, el cual debe ser respondido por los cuidadores de los niños menores de 10 años ${ }^{11}$. De los cuatro, CAN es el único cuestionario validado en español, cuenta con dos versiones, una para niños de 2 a 8 años con preguntas que deben ser respondidas por el cuidador y otra desde los 9 hasta los 14 años, que debe responder el niño/adolescente o cuidador ${ }^{12}$.

El objetivo de este estudio fue comparar las versiones validadas en español de CAN con las recomendaciones de control de asma de GINA, las cuales fueron consideradas como referencia.

\section{Material y Método}

El estudio fue realizado en el policlínico de enfermedades respiratorias del hospital de niños Roberto del Río, entre marzo de 2010 y febrero de 2011. Cada día se enroló de forma no aleatoria a uno o dos niños asmáticos de 5 a 14 años desde la sala de espera del laboratorio de función pulmonar, al cual asistieron para realizar una espirometría de control. Se aplicó por medio de una entrevista, el cuestionario de control de síntomas de asma sugerido por GINA para pacientes asmáticos mayores de 5 años en su versión en español disponible en el sitio web: www.ginasthma.org. Entre los 5 y 8 años el cuestionario GINA fue respondido por los cuidadores. En el grupo de 9 a
14 años lo hicieron los niños o adolescentes. Este cuestionario clasifica el control del asma en los últimos siete días en tres niveles: asma controlada, parcialmente controlada y no controlada. Esto nos permitió dividir la muestra en dos grupos: un grupo de pacientes controlados y otro que incluyó parcialmente controlados y no controlados.

Con previa autorización de los autores, se aplicó a niños y cuidadores el cuestionario $\mathrm{CAN}^{12}$. Las versiones en español fueron obtenidas del sitio web http://www.respirar.org/calidadvida/can. $\mathrm{htm}$. Los cuidadores de los niños entre 5 y 8 años, respondieron la versión CAN tutor y los niños o adolescentes de 9 a 14 años la versión CAN niño/ adolescente. El cuestionario CAN, que es autoadministrable, fue diseñado para medir el control de asma en el último mes. Ambas versiones de CAN cuentan con 9 preguntas, cada una con 5 posibles respuestas en una escala ordinal de Likert desde 0 puntos (ausencia de síntomas) hasta 4 puntos (máxima sintomatología). Se obtiene un puntaje por cada pregunta y un puntaje promedio total con un rango que va de 0 puntos (mejor control) a 36 puntos (peor control). El cuestionario cataloga como no controlados a los pacientes con puntaje promedio total igual o superior a 8 puntos y controlados a los que obtienen puntajes menores de 8 puntos.

Las entrevistas fueron realizadas por un médico familiarizado con las encuestas. Se obtuvo autorización del cuidador para aplicar ambas encuestas mediante un consentimiento informado. El mismo día, todos los pacientes realizaron una espirometría, registrándose el $\mathrm{VEF}_{1}$ (porcentaje del predicho), valor exigido por la recomendación GINA para completar la evaluación del control de asma. La espirometría fue realizada por un médico especializado en estudio respiratorio funcional, con un espirómetro Jaeger modelo MS-PFT, utilizando los valores predictivos de Knudson et al. Se excluyeron a aquellos asmáticos que cursaron una infección intercurrente en las últimas 2 semanas. Se midió concordancia e índice Kappa entre control obtenido por las versiones de CAN y GINA. Además se calculó la sensibilidad, especificidad, valor predictivo positivo, valor predictivo negativo para las dos versiones de CAN, utilizando a GINA como referencia. El tamaño muestral fue calculado con un poder de $80 \%$ y valor de $\mathrm{p}<0,05$. Para los cálculos estadísticos se utilizó el programa SPSS.

\section{Resultados}

Enrolamos a 214 niños asmáticos persistentes. Género masculino 60,3\%. Promedio de edad 8,3 
Tabla 1. Porcentaje de síntomas de asma según cuestionario CAN*

\begin{tabular}{|lcc|}
\hline Síntomas & $\begin{array}{c}\text { CAN Tutor } \\
\mathbf{( \% )}\end{array}$ & $\begin{array}{c}\text { CAN niño/adolescente } \\
(\mathbf{\%})\end{array}$ \\
\hline Tos diurna & 84 & 72 \\
Tos nocturna & 75 & 66 \\
\hline Sibilancias diurnas & 36 & 41 \\
Sibilancias nocturnas & 39 & 34 \\
\hline Dificultad para respirar en el día & 36 & 50 \\
Dificultad para respirar en la noche & 46 & 35 \\
Tos con ejercicio, juego o risa a carcajadas & 80 & 85 \\
Visitas a urgencia por crisis de asma & 34 & 37 \\
\hline Hospitalización mayor a 12 h por crisis de asma & 9 & 16 \\
\hline
\end{tabular}

*CAN: cuestionario de control de asma en niños.

Tabla 2. Concordancia entre control de asma medido según recomendación GINA y dos versiones de cuestionario CAN*

\begin{tabular}{|lccc|}
\hline Cuestionario & $\begin{array}{c}\text { Diagnóstico acertado } \\
\mathbf{( \% )}\end{array}$ & $\begin{array}{c}\text { Kappa } \\
\text { IC 95\% }\end{array}$ & p \\
CAN tutor & 76,5 & 0,4 & 0,000000002 \\
& & $(0,2-0,5)$ & 0,000000005 \\
CAN niño/adolescente & 79,2 & 0,55 & $(0,4-0,7)$ \\
\end{tabular}

*CAN: cuestionario de control de asma en niños. IC: intervalo de confianza.

años. La distribución de severidad según GINA fue la siguiente: $42(19,6 \%)$ persistentes leves, $127(59,4 \%)$ persistentes moderados y 45 (21\%) persistentes severos.

Todos los niños y tutores fueron capaces de responder los cuestionarios. El promedio de tiempo en contestar la encuesta fue de 4,3 min para los tutores y 5,2 min para los niños/adolescentes. Todos los niños o tutores lograron entender las preguntas y sólo casos aislados requirieron la explicación de alguna pregunta puntual.

Los cuidadores de niños entre 5 y 8 años reportaron $12,1 \%$ de asma controlada con el cuestionario GINA y 35,6\% con la versión CAN tutor.

En el grupo de niños de 9 a 14 años, la proporción de asmáticos controlados alcanzó el 23,2\% con GINA y $43,9 \%$ con CAN niño/adolescente.

La Tabla 1 muestra la distribución porcentual de los síntomas de asma encontrados con el cuestionario CAN en ambos rangos de edad, destacando a la tos diurna, nocturna o con ejercicio como la sintomatología más frecuentemente encontrada en ambos rangos de edad.

El promedio de $\mathrm{VEF}_{1}$ fue $109 \%$ y sólo el 3,7\% de la serie (ocho pacientes), registraron valores inferiores al $80 \%$.

El grado de acuerdo entre las encuestas fue elevado llegando casi al $80 \%$ con ambas versiones de CAN, con un índice de concordancia moderado, que fue ligeramente superior para cuestionario CAN dirigido a niños de 9 a 14 años que a los menores de 9 años. El porcentaje de acuerdo entre el control obtenido por CAN y GINA con sus respectivos índices de Kappa se representan en la Tabla 2. Las sensibilidad, especificidad, valor predictivo positivo y valor predictivo negativo fueron las pruebas diagnósticas evaluadas en las dos versiones del cuestionario CAN (Tabla 3 ).

\section{Comentarios}

El cuestionario de control GINA fue diseñado por un grupo de expertos para ser utilizado en pacientes asmáticos mayores de 12 años y adultos $^{13,14}$. El grado de conocimiento y la difusión de este cuestionario en los pediatras especialistas en enfermedades respiratorias, fueron las razones para considerarlo como la referencia en nuestro estudio. Sin embargo, se debe mencionar que este 
Tabla 3. Pruebas diagnósticas para control de asma en niños medido por cuestionario de control de asma en niños (CAN)

\begin{tabular}{|lcccc|}
\hline Cuestionario & $\begin{array}{c}\mathbf{S} \\
(\mathbf{I C ~ 9 5 \% )}\end{array}$ & $\begin{array}{c}\mathbf{E} \\
(\mathbf{I C ~ 9 5 \% )}\end{array}$ & $\begin{array}{c}\text { VP (+) } \\
(\mathbf{I C} \text { 95\%) }\end{array}$ & $\begin{array}{c}\text { VP (-) } \\
\text { (IC 95\%) }\end{array}$ \\
CAN tutor & $100 \%$ & $73 \%$ & $34 \%$ & $100 \%$ \\
& $(76-99)$ & $(64,1-80,9)$ & $(21,2-49,4)$ & $(94,6-99,9)$ \\
CAN niño/adolescente & $100 \%$ & $73 \%$ & $53 \%$ & $100 \%$ \\
& $(79,1-99,5)$ & $(60,1-83,1)$ & $(35,7-69,2)$ & $(90,4-99,8)$ \\
\hline
\end{tabular}

S: sensibilidad, E: especificidad, VP (+): valor predictivo positivo, VP (-): valor predictivo negativo, IC: intervalo de confianza.

cuestionario no cuenta con una validación formal en asmáticos menores de 12 años.

En esta investigación se demostró que los niños asmáticos y sus tutores son capaces de responder sin dificultad las dos versiones del cuestionario CAN en un tiempo muy similar a los cuatro minutos de la versión original.

En dos series de asmáticos adolescentes mayores de 12 años y adultos, se comparó la versión en inglés de ACT con GINA, obteniendo grados de acuerdo y concordancia variables, dependiendo de la población estudiada ${ }^{15,16}$. En menores de 12 años, un estudio realizado con niños de 4 a 11 años, notificó un $26 \%$ de discrepancia entre C-ACT y GINA para el diagnóstico de asma controlada ${ }^{17}$.

Nuestro estudio encontró un bajo porcentaje de desacuerdo entre CAN y GINA, que como máximo alcanzó el 23,5\% y una concordancia moderada según el índice de Kappa, lo que es clínicamente relevante y aceptable del punto de vista estadístico. Sin embargo, en ambos grupos etareos se obtuvo un mayor porcentaje de asma controlada con CAN que con el cuestionario GINA. Esto debería ser analizado con detención, ya que podría sugerir una menor capacidad de CAN para diagnosticar a los pacientes controlados. En estudios con un diseño similar a este, en los cuales se comparó el cuestionario C-ACT con GINA en menores de 12 años, se concluyó que C-ACT tiende a sobrestimar el control del asma, es decir a catalogar como controlados a algunos pacientes no controlados por GINA, por lo que se ha recomendado elevar el punto de cohorte que tiene la encuesta para clasificar a los pacientes como controlados ${ }^{18,19}$.

Otro hallazgo importante fue que para el grupo de 5 a 8 años, ambas encuestas detectaron una menor proporción de pacientes controlados que en el grupo de pacientes de 9 a 14 años. Esto podría ser explicado porque en el grupo de menor edad ambas encuestas fueron respondidas por los padres o tutores, quienes tienden a ser más estrictos e incluso a veces subestiman el control de la enfermedad, a diferencia de los adolescentes quienes interpretan con mayor dificultad los síntomas de mal control o lo sobrestiman. Respecto a este punto, la literatura mantiene controversias, ya que algunos autores destacan la importancia de la percepción de control parental en desmedro de la del niño en los menores de 11 años. Sin embargo, otros sugieren considerar la discrepancia entre la percepción del control del niño y su cuidador ${ }^{20-23}$.

El grado de validez del cuestionario CAN se midió a través de la sensibilidad y especificidad. La sensibilidad de CAN se definió como la probabilidad de catalogar correctamente controlados a los asmáticos controlados por GINA. La capacidad de CAN para diagnosticar correctamente como no controlados a los pacientes no controlados por GINA, se definió como especificidad de la prueba. Tanto la sensibilidad como la especificidad de las versiones de los cuestionarios CAN fueron bastante aceptables, incluso mejores que la reportada en la versión original y sus réplicas en España ${ }^{12,24}$.

Esto es importante, ya que otros cuestionarios como el C-ACT, que mide el control de asma en niños de 4 a 11 años, incorporando la percepción del control del niño por medio de una escala visual análoga, no ha logrado buenos niveles de sensibilidad o especificidad cuando ha sido comparado con GINA ${ }^{25}$.

La seguridad de la prueba se midió por medio de los valores predictivos. El valor predictivo positivo fue definido como la probabilidad de que un niño asmático con un valor de $\mathrm{CAN}<8$ puntos (controlado), fuese clasificado como controlado según la recomendación GINA. Ambas versiones de CAN mostraron bajos valores predictivos positivos. Sólo un tercio de los pacientes controlados por CAN tutor y la mitad de los controlados por CAN niño/adolescente, fueron 
correctamente clasificados como controlados de acuerdo a GINA, es decir, diagnosticar a un niño o adolescente asmático como controlado no necesariamente significa que este realmente lo sea, siendo más difícil a medida que el paciente tiene menor edad. Por el contrario, el valor predictivo negativo correspondió a la probabilidad de que un niño con CAN $\geq 8$ puntos (no controlado) fuese efectivamente catalogado como no controlado por GINA. El valor predictivo negativo para ambas versiones de CAN llegó al máximo. El análisis de los valores predictivos permite concluir que ambas versiones de CAN son cuestionarios útiles para detectar con exactitud a los niños o adolescentes asmáticos que no llevan un buen control de la enfermedad, pero menos efectivos para identificar a los niños o adolescentes verdaderamente controlados, de acuerdo a la referencia utilizada.

A pesar de que este estudio notificó buena concordancia y validez de CAN con respecto a un patrón de referencia como GINA, no cumple con todos los requisitos necesarios para un estudio de validación. Existen al menos tres criterios exigidos para la validación de cuestionarios clínicos que no fueron incorporados en la metodología de esta investigación: la fiabilidad o consistencia interna de la prueba, la estabilidad temporal del cuestionario, que debe ser medida por el mismo entrevistador en dos tiempos distintos y la adaptación cultural del cuestionario en la población aplicada, aunque la versión original tenga el mismo idioma que la población en la cual fue aplicado $^{26-29}$.

Finalmente podemos concluir que el cuestionario CAN es otra alternativa disponible para medir el control del asma en niños o adolescentes de nuestro medio, el cual puede ser aplicado rápidamente $\mathrm{y}$ con fácil entendimiento por parte de los pacientes y sus cuidadores. A su vez, logra una concordancia, validez y seguridad aceptables al ser comparado con las recomendaciones de control sugeridas por GINA, especialmente en el diagnóstico del asma no controlada.

\section{Bibliografía}

1.- GLOBAL INITIATIVE FOR ASTHMA, GLOBAL STRATEGY FOR ASTHMA MANAGEMENT AND PREVENTION. Diagnosis and classification. Chapter 2. Updated 2010. Available at: http:// www.ginasthma. org [Consultado el 24 de marzo de 2012].

2.- REDDEL H, TAYLOR D, BATEMAN E, BOULET L, BOUSHEY H, BUSSE W, et al. An official American Thoracic Society/European Respiratory Society state- ment: asthma control and exacerbations: standardizing endpoints for clinical asthma trials and clinical practice. Am J Respir Crit Care Med 2009; 180: 59-99.

3.- NATIONAL ASTHMA EDUCATION AND PREVENTION PROGRAM. Expert Panel Report 3 (EPR-3): Guidelines for the Diagnosis and Management of Asthma-Summary Report 2007. J Allergy Clin Immunol 2007; 120: S94-138.

4.- $\quad$ NATHAN R, SORKNESS C, KOSINSKI M, SCHATZ M, LI J, MARCUS P, et al. Development of the asthma control test: a survey for assessing asthma control. J Allergy Clin Immunol 2004; 113: 59-65.

5.- VEGA J, BADIA X, BADIOLA C, LÓPEZ-VIÑA A, OLAGUÍBEL J, PICADO C, et al. Validation of the Spanish version of the Asthma Control Test (ACT). J Asthma 2007; 44: 867-72.

6.- JUNIPER E, O'BYRNE P, GUYATT G, FERRIE P, KING D. Development and validation of a questionnaire to measure asthma control. Eur Respir J 1999; 14: 902-7.

7.- PICADO C, BADIOLA C, PERULERO N, SASTRE J, OLAGUÍBEL J, LÓPEZ VIÑA A, et al. Validation of the Spanish version of the Asthma Control Questionnaire. Clin Ther 2008; 30: 1918-31.

8.- SKINNER E, DIETTE G, ALGATT-BERGSTROM P, NGUYEN T, CLARK R, MARKSON L, et al. The Asthma Therapy Assessment Questionnaire (ATAQ) for children and adolescents Dis Manag 2004; 7: 305-13.

9.- DIETTE G, SAJJAN S, SKINNER E, WEISS T, WU A, MARKSON L. Using the Pediatric Asthma Therapy Assessment Questionnaire to Measure Asthma Control and Healthcare Utilization in Children. Patient 2009; 2: 233-41.

10.- LIU A, ZEIGER R, SORKNESS C, MAHR T, OSTROM N, BURGESS S, et al. Development and crosssectional validation of the Childhood Asthma Control Test. J Allergy Clin Immunol 2007; 119: 817-25.

11.- JUNIPER E, GRUFFYDD-JONES K, WARD S, SVENSSON K. Asthma Control Questionnaire in children: validation, measurement properties, interpretation. Eur Respir J 2010; 36: 1410-6.

12.- PÉREZ-YARZA E, BADÍA X, BADIOLA C, COBOS N, GARDE J, IBERO M, et al. Development and Validation of a Questionnaire to Assess Asthma Control in Pediatrics. Pediatric Pulmonology 2009; 44: 54-63.

13.- BATEMAN E, BOUSQUET J, BAUNSTEIN G. Is overall asthma control being achieved? A hypothesisgenerating study. Eur Respir J 2001; 17: 589-95.

14.- BATEMAN E, HURD S, BARNES P, BOUSQUET J, DRAZEN J, FITZGERALDE M, et al. Global strategy for asthma Management and prevention: GINA executive summary Eur Respir J 2008; 31: 143-78.

15.- THOMAS M, KAYB S, PIKEB J, WILLIAMSC A, ROSENZWEIG J, HILLYERD PRICE D, et al. The Asthma Control TestTM (ACT) as a predictor of GINA guideline-defined asthma control: analysis of a multina- 
tional cross-sectional survey. Primary Care Respiratory Journal 2009; 18: 41-9.

16.- NGUYEN V, CHAVANNES N, LE L. PRICE D. The Asthma Control Test (ACT) as an alternative tool to Global Initiative for Asthma (GINA) guideline criteria for assessing asthma control in Vietnamese outpatients. Prim Care Respir J 2012; 21: 85-9.

17.- ERKOÇOĞLU M, AKAN A, CIVELEK E, KAN R, AZKUR D, KOCABAŞ C. Consistency of GINA criteria and childhood asthma control test on the determination of asthma control. Pediatr Allergy Immunol 2012; 23: $34-9$

18.- YU H, NIU C, KUO H, TSUI K, WU C, KO C, et al. Comparison of the Global Initiative for Asthma guideline-based Asthma Control Measure and the Childhood Asthma Control Test in evaluating asthma control in children. Pediatr Neonatol 2010; 51: 273-8.

19.- ITO Y, ADACHI Y, ITAZAWA T, OKABE Y, ADACHI Y, HIGUCHI O, et al. Association between the Results of the Childhood Asthma Control Test and Objective Parameters in Asthmatic Children J Asthma 2011; 48: 1076-80.

20.- GUYATT G, JUNIPER E, GRIFFITH L, FEENY D, FERRIE P. Children and Adult Perceptions of Childhood Asthma. Pediatrics 1997; 99: 165-68.

21.- YOOS H, KITZMAN H, MCMULLEN A, SIDORA K. Symptom perception in childhood asthma: how accurate are children and their parents?. J Asthma 2003; 40: 27-39.
22.- DOZIER A, ALIGNE C, SCHLABACH M. What is asthma control? Discrepancies between parents' perceptions and official definitions. J Sch Health 2006; 76 : 215-8.

23.- DELL S, FOTY R, BECKER A, FRANSSEN E, CHAPMAN K. Parent-reported symptoms may not be adequate to define asthma control in children. Pediatr Pulmonol 2007; 42: 1117-24.

24.- CANO A, UREÑA I, DÍAZ C, MORA I, MOLA P, GARCÍA A, et al. Control del asma en niños: validez del cuestionario CAN y su relación con la función pulmonar y el óxido nítrico exhalado. Bol Pediatr 2010; 51 : $39-46$.

25.- KOOLEN B, PIJNENBURG M, BRACKEL H, LANDSTRA A, VAN DEN BERG N, MERKUS P, et al. Comparing Global Initiative for Asthma (GINA) criteria with the Childhood Asthma Control Test (C-ACT) and Asthma Control Test (ACT). Eur Respir J 2011; 38: 561-6.

26.- GUYATT G. Users' Guides to the Medical Literature. American Medical Association. 2002.

27.- BIME C, NGUYEN J, WISE R. Measures of asthma control. Curr Opin Pulm Med 2012; 18: 48-56.

28.- JUNIPER E. Medical Questionnaires Are Copyrighted To Ensure That Validity Is Maintained. Chest 2009; 136 : 951-52.

29.- JUNIPER E. Validated questionnaires should not be modified. Eur Respir J 2009; 34: 1015-17.

Correspondencia a:

Dr. Alberto Vidal G.

Pediatra Especialista en Enfermedades Respiratorias

Pediátricas

Dirección: Holanda 87 Dpto. 604 Providencia /Stgo.

E-mail: aevgmd@yahoo.es 